\title{
Offene Bildungsmaterialien als Wegbereiter zu einer Kultur des Teilens in der Erwachsenen- und Weiterbildung - Befunde aus Sicht der Lehrenden
}

\author{
Sabine Schöb $(\mathbb{D} \cdot$ Carmen Biel $\cdot$ Lars Kilian
}

Eingegangen: 5. März 2021 / Überarbeitet: 29. Juni 2021 / Angenommen: 22. Juli 2021 / Online publiziert: 20. August 2021

(C) Der/die Autor(en) 2021

Zusammenfassung Der Beitrag thematisiert Herausforderungen bei der Etablierung einer Community von Lehrenden der Erwachsenen- und Weiterbildung, die durch den freien und inkludierenden Austausch von Offenen Bildungsmaterialien (Open Educational Resources, OER) geprägt ist. Aus der Sicht der Lehrenden wird betrachtet, welche Bedeutung vorhandene Kompetenzen, persönliche Einstellungen und soziale Werthaltungen Lehrender für den Umgang mit OER haben und welche Rahmenbedingungen für einen aktiven und kollaborativen Umgang im Sinne einer Open Culture förderlich sind. Die über eine quantitative Befragung gewonnenen Aussagen werden mit einer Auswertung der Nutzung von Informations- und Lernangeboten zum sachgerechten Umgang mit OER am Beispiel des Portals wb-web gerahmt. Die Ergebnisse zeigen vor allem den Bedarf, rechtliche Sicherheit und Infrastrukturen zu schaffen, um das Erstellen, Teilen und Nachnutzen von qualitativ hochwertigen und bedarfsgerecht einsetzbaren OER zu ermöglichen.

Schlüsselwörter Offenheit in der Erwachsenen- und Weiterbildung · Open Educational Resources · Open Culture

\footnotetext{
Sabine Schöb

Universität Tübingen, Tübingen, Deutschland

E-Mail: sabine.schoeb@uni-tuebingen.de

Carmen Biel $(\bowtie) \cdot$ Lars Kilian

Deutsches Institut für Erwachsenenbildung, Bonn, Deutschland

E-Mail: biel@die-bonn.de

Lars Kilian

E-Mail: kilian@die-bonn.de
} 


\title{
Open educational resources as enablers for a culture of sharing in adult and continuing education-Findings from the perspective of teachers
}

\begin{abstract}
The article addresses the challenges of establishing a community of teachers in adult and continuing education that is characterized by the free and inclusive exchange of Open Educational Resources (OER). From the perspective of the teachers, the importance of existing competencies, personal attitudes and social values of teachers for the handling of OER and which framework conditions are conducive to an active and collaborative handling in the sense of an Open Culture will be considered. The statements obtained via a quantitative survey are framed with an evaluation of the use of information and learning offers for the appropriate handling of OER using the example of the portal wb-web. The results show above all the need to create legal security and infrastructures to enable the creation, sharing and reuse of high-quality OER that can be used according to needs.
\end{abstract}

Keywords Openness in Adult and Continuing Education · Open Educational Resources · Open Culture

\section{Ausgangslage}

Eine Open Education mit dem Ziel der Flexibilisierung von Lehr-Lernmöglichkeiten und der Erhöhung der gesellschaftlichen Teilhabe bedarf einer Verfügbarkeit von offenen Bildungsmaterialien sowie der Bereitschaft Lehrender, diese Materialien zu erstellen, weiterzugeben und nachzunutzen. Herausforderungen bestehen dabei nicht nur bezüglich der inhaltlich-formalen Erstellung von qualitativ hochwertigen und rechtlich abgesicherten Bildungsmaterialien, auf die Nachnutzende zugreifen können. Vielmehr steht und fällt Open Education mit der aktiven Beteiligung der Lehrenden als Produzierende und Nachnutzende von Open Educational Resources (OER) und der Etablierung einer Kultur des Teilens in der Erwachsenen- und Weiterbildung (vgl. Powell 2015, S. 377). Mit Blick auf die für eine Open Culture zu motivierenden Lehrenden sowie deren Vernetzung sind die verhaltensbedingenden sachlichen, persönlichen und sozialen Dimensionen des Könnens, Wollens und Dürfens (vgl. von Rosenstiehl et al. 2005, S. 259) betrachtungsrelevant, um Ansatzund Unterstützungsmöglichkeiten für einen durch gemeinsame Zusammenarbeit und wechselseitige Unterstützung gekennzeichneten Umgang mit OER zu identifizieren.

Hier setzt der Beitrag an. Er thematisiert die Nutzung von OER in der Weiterbildungspraxis und fragt nach Erfolgsfaktoren für die Etablierung einer Kultur des Teilens bei Lehrenden der Erwachsenen- und Weiterbildung. Den Ausgangspunkt bildet das Portal wb-web (wb-web.de), das neben Informations- und Vernetzungsangeboten auch freie Bildungsressourcen zur Gestaltung von Lehr-Lernsituationen sowie eine Lernumgebung mit sequenzierten Lernpfaden zur Kompetenzentwicklung Lehrender u. a. im Umgang mit OER (EULE Lernbereich, wb-web.de/lernen.html) anbietet. 


\section{Theoretische Grundlagen und wissenschaftlicher Diskussionsstand}

Im erziehungswissenschaftlichen Diskurs der letzten Jahre gibt es laut Kerres (2019, S. 2) zahlreiche Stränge, die sich mit dem Thema Offenheit beschäftigen. Versucht man diese in eine hierarchische Logik zu bringen, lassen sich Open Source, Open Standards und OER als untergeordnete Ansätze begreifen, die Merkmale und Elemente der Gestaltung von Open Education thematisieren (vgl. Kerres 2019, S. 4 f.). Open Education selbst sowie Open Culture spannen als übergeordnete Ansätze die Grundidee und den Rahmen einer „freien Bildung für Alle“ auf.

„Open Education“ kann als Überbegriff für „Offenheit der Bildung“ gefasst werden (vgl. Blees et al. 2015, S. 53; Deimann 2019, S. 5), der ein „rethinking the numerous dimensions that are familiar in traditional educational structures and patterns“ (Ossiannilsson et al. 2016, S. 160) erfordert. „Offen“ ist dabei der zentrale Faktor:

Open is key; open allows not just access, but the ability to modify and use materials, information and networks so education can be personalized to individual users or woven together in new ways for large and diverse audiences. (Open Education Consortium o.J.)

So verbindet Open Education die Idee des Wissensaustauschs und der -genese mit modernen Technologien, um einen großen Pool offener, geteilter sowie bedarfsgerechter Bildungsressourcen zu schaffen und den Geist der Zusammenarbeit - den „spirit of collaboration“ - dafür zu nutzen (vgl. Open Education Consortium o.J.).

Um den Ansatz der Open Education zu beleben, bedarf es Open Educational Resources (OER). OER sind Bildungsmaterialien jeglicher Art und in jedem Medium, die unter einer offenen Lizenz veröffentlicht werden (vgl. UNESCO 2015, S. 6; Deimann 2019, S. 2f.). Eine solche offene Lizenz ermöglicht den kostenlosen Zugang sowie die kostenlose Nutzung, Bearbeitung und Weiterverbreitung durch Andere ohne oder mit Einschränkungen (vgl. Blees et al. 2015, S. 12).

Produzierende können neben Verlagen und Bildungsanbietern insbesondere die Lehrenden selbst sein. Bei der Erstellung von OER finden traditionelle akademische und wissenschaftliche Qualitätssicherungsverfahren durch Peer Reviews und externe Bewertung häufig keine Anwendung, was - wie vorliegende Studien zeigen - besondere Herausforderungen für die Produktion und die Verwendung der Materialien mit sich bringt sowie mit einem Mangel an Vertrauen in offene Bildungsmaterialien einhergeht (vgl. Camilleri und Ehlers 2011, S. 9). Die bloße Verfügbarkeit von OER lässt nicht auf deren Qualität, den didaktischen Mehrwehrt und die tatsächlichen Einsatzszenarien schließen (vgl. Mayrberger und Hofhues 2013, S. 57). Für den Einsatz von qualitativ hochwertigen OER Inhalten und Formaten bedarf es vielmehr didaktischen und technischen Know-hows seitens der Erstellenden und der Nachnutzenden (vgl. Mayrberger und Hofhues 2013, S. 61). Auch rechtliche Fragen, z. B. hinsichtlich des Urheberrechts oder der Lizenzierung von OER, aber auch bei der Nutzung fremderstellter Inhalte für eigene (freie) Bildungsressourcen müssen geklärt werden (vgl. Mayrberger und Hofhues 2013, S. 61). Bei den Nutzenden wiederum bestehen Bedenken, insbesondere hinsichtlich der Qualität, und Abwehrreaktionen beim Einsatz von OER in der Lehre (vgl. z.B. Deimann und Bastiaens 
2010, S. 15; Ehlers 2016, S. 96; Zawacki-Richter und Mayrberger 2017, S. 18), denen es für eine Verbreitung von OER in der Weiterbildungspraxis zu begegnen gilt. Es lassen sich nach Weller et al. (2016) Lehrende unterscheiden, die (1) OER passiv (aber breit) für die Gestaltung von Lehrangeboten verwenden, (2) OER nur als (gelegentlichen) Ergänzungsbaustein für die eigenen Lehrkonzepte verwenden oder (3) OER selbst produzieren und sich für deren Nachnutzung einsetzen. Mögliche Erklärungen für den unterschiedlichen Umgang bestehen in den persönlichen Voraussetzungen und vorhandenen Ressourcen der Lehrenden, der Sicherheit bezüglich des möglichen Umgangs mit OER und der Eingebundenheit in sich gegenseitig stützende Communities (vgl. Weller et al. 2016, S. $80 \mathrm{ff}$.). Insgesamt betrachtet liegen jedoch noch wenig Befunde zu der empirischen Untersuchung des Potenzials von OER zur Förderung einer Open Culture in der Bildungspraxis im Allgemeinen und der Erwachsenenbildung im Speziellen vor (vgl. Ehlers 2013, S. 91). Zugleich ist die Gruppe der in diesem Berufsfeld Lehrenden durch eine hohe Heterogenität in ihren persönlichen Hintergründen, ihrer beruflichen Lage und ihren konkreten Handlungskontexten gekennzeichnet (vgl. Martin et al. 2016), die eine besondere Herausforderung für die Etablierung einer durch gemeinsame Werte und übergreifende Standards getragenen Community darstellt.

Genau darin besteht jedoch eine Grundanforderung für eine Verbreitung des Einsatzes von OER. Produzierende und Nutzende von OER benötigen ,a change in attitudes and mindset that emphasizes flexible growth instead of fixed traditions" (Ossiannilsson et al. 2016, S. 163), der auf eine Kultur der Offenheit angelegt und durch die Bereitschaft zum Teilen gekennzeichnet ist. Es geht darum, Produzierende mit Nutzenden in einer nicht kommerziell motivierten Community zu vereinen, in der sie kollaborativ zusammenwirken und zur Erhöhung der Zugänglichkeit und Verbreitung der Nutzung freier Materialien beitragen, was durch bestimmte - idealerweise gemeinsam von den Community-Mitgliedern geteilte - Werte, Weltanschauungen und Grundhaltungen begünstigt wird (vgl. Friesinger et al. 2015, S. 9 ff.). Das einer Open Culture zugrundeliegende Prinzip des freien und inkludierenden Informations- und Wissensaustausches bedarf einer offenen Einstellung der Beteiligten, die u.a. durch Großzügigkeit und die Bereitschaft zu Geben gekennzeichnet ist und die Prozesse und Strukturen des Einsatzes von OER in der Bildungspraxis stärkt (vgl. Wiley 2010, S. 16).

Je heterogener die Voraussetzungen und Handlungsbedarfe der Beteiligten sind, umso vielfältiger sind jedoch die Ansprüche und Erwartungen an OER, die es zur Etablierung einer gelebten Open Culture zu integrieren bzw. zu tolerieren gilt (vgl. Powell 2015, S. 379f.). Diese rekrutieren auf (a) sachliche, (b) persönliche und (c) soziale Dimensionen der Produktion und der Nutzung von OER, wie die Fähigkeit Lehrender, OER zu erstellen und nachzunutzen, die Haltung der Lehrenden gegenüber OER sowie die sozialen Werte im Umgang mit OER.

Diese drei Dimensionen sollen im weiteren Verlauf der Darstellung genauer betrachtet werden, da sie den Gegenstand der dem Beitrag zugrunde gelegten Untersuchung zur Bewertung des Potenzials von OER für die Weiterbildungspraxis aus Sicht der Lehrenden in der Erwachsenenbildung bildeten. Die Zuordnung der Aspekte je Dimension erfolgt basierend auf den psychologischen Modellannahmen des Verhaltens als Produkt der Fähigkeit und der Motivation von Personen im situa- 
Abb. 1 Verhaltensbedingende Dimensionen. (Eigene Darstellung)

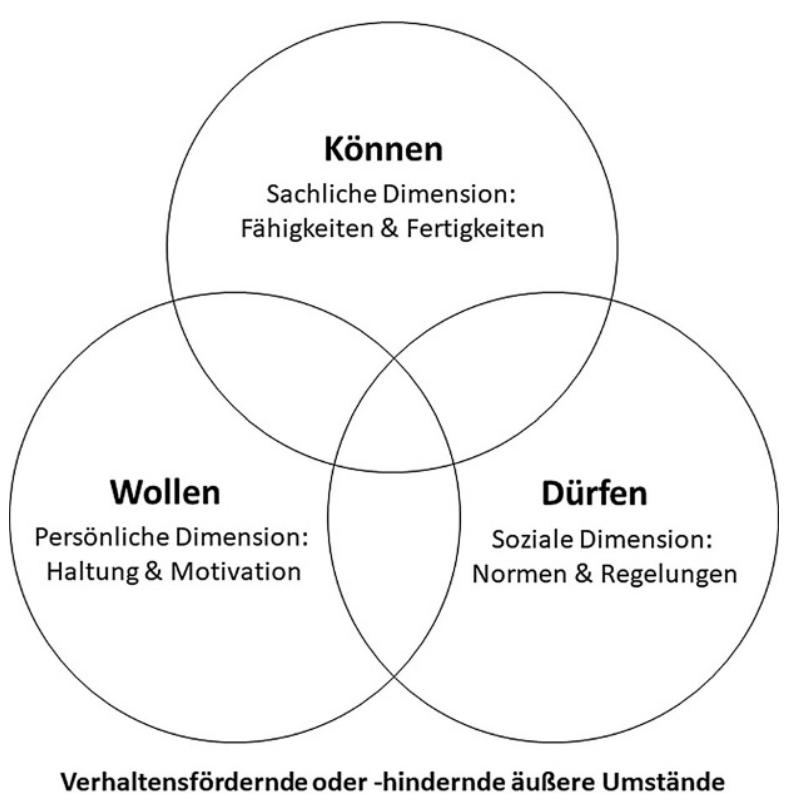

tiven, durch soziale Normen und Regelungen bestimmten Kontext (vgl. von Rosenstiehl et al. 2005, S. 259). Dabei stehen die einzelnen Dimensionen des Verhaltens in Wechselwirkung zueinander und weisen Schnittmengen zueinander auf, so zum Beispiel bezüglich der Bereitschaft Lehrender OER zu teilen, die von persönlichen Aspekten wie eigenen Haltungen und Motiven ebenso abhängig ist wie von sozialen Normen und Regelungen der Communities, in denen ein aktiver Informations- und Wissensaustausch zur Nutzung von OER etabliert werden soll. Weiter werden die Verhaltensmöglichkeiten der Einzelnen durch hemmende oder begünstigende äußere Umstände auf institutioneller bzw. gesellschaftlicher Ebene mitbedingt (vgl. Abb. 1).

\subsection{Sachliche Dimension - Können}

Die sachliche Dimension adressiert insbesondere die Qualität von OER und wird durch den sachgerechten, gekonnten Umgang der Produzierenden und Nachnutzenden mit OER bestimmt.

Ohne Kenntnisse über die „Funktionsweise“ von OER, wie z.B. der Lizenzierung, können OER nicht ihren Charakteristika entsprechend erstellt werden (vgl. Braun 2008, S. 45, 66). Greifen Produzierende nicht auf entsprechende Lizenzen zurück, sind OER aufgrund einer unklaren rechtlichen Rahmung schlichtweg nicht nutzbar. Auch für die Entscheidung bei der medialen Erstellung - und hier insbesondere bei der Wahl des Ablageformats - ist OER-spezifisches Wissen nötig. Wird die Lernressource zwar offen lizenziert und zur Weiterbearbeitung freigegeben, sind auch entsprechend offene Dateiformate nötig. Ein geschlossenes Format trägt nicht zur Verbesserung der Qualität seitens der Nachnutzenden bei. Denn laut der am Projekt OER-MuMiW (www.oer-fachexperten.de/projekt) beteiligten Leh- 
renden Sabine Preusse ist „,der vielleicht wichtigste Aspekt zur Qualität von OER [...] die Zusammenarbeit an Materialien (BMBF 2020)“.

Neben dem auf rechtliche und technische Fragen bezogenen Wissen zu OER gehört auch pädagogisches - konkreter - didaktisches Wissen. Dieses ist für die inhaltliche Seite der Ressourcen zentral, die zunächst passgenau für die Bedarfe und Settings der Erstellenden aufbereitet werden müssen. Während dies eine herkömmliche didaktische Aufgabe Lehrender bei der Erstellung von Bildungsmaterialien zur Vermittlung zwischen der Sachlogik des Inhalts und der Psychologik des/der Lernende/n darstellt (vgl. Siebert 2019, S. 10), kommt bei der Bereitstellung dieser als OER die Herausforderung dazu, dass die erfolgte didaktische Transformation auf ein konkretes Trainingssetting (vgl. Aschersleben 1993, S. 91-106) als Metainformation mitgeliefert werden sollte, damit eine Dekontextualisierung und erneute Rekontextualisierung für die eigenen Bedarfe und Settings für die Nachnutzenden möglich wird (vgl. Siebert 2019, S. 212 ff.).

Auf der anderen Seite stehen die Nachnutzenden der OER vor der nicht unerheblichen Herausforderung, für eigene Kurse entsprechendes Material überhaupt erst zu finden und dessen Aktualität zu überprüfen. Sie müssen in der Lage sein, OER auf Basis der verwendeten Lizenzen auszuwählen, um diese für eigene Trainings rechtssicher einsetzen zu können. Darüber hinaus benötigen sie entsprechende Kompetenzen, um mit den abgelegten Inhalten und Formaten umzugehen (vgl. Mayrberger und Hofhues 2013, S. 62). Diese dürften in der Regel keine volle Passung für das eigene Kurskonzept haben und müssen für eine Nachnutzung adaptiert werden. Dies ist nicht nur für den technisch funktionierenden Einsatz in den eigenen LehrLern-Settings bedeutsam, sondern auch für die erneute Veröffentlichung der dabei rekombinierten Inhalte als OER.

\subsection{Persönliche Dimension - Wollen}

Die persönliche Dimension zielt auf zwei Bereiche: die persönliche Einstellung zu sowie die eigenen Erfahrungen mit OER als Nutzende und auch als Produzierende. Otto (2020, S. 78 f.) betont, dass vorhandene, durch positive sowie negative Gefühle verstärkte Einstellungen und Motive Faktoren sind, die sich maßgeblich auf das Verhalten Lehrender im Umgang mit OER auswirken.

Die Sorge um die eigene Reputation kann mit Blick auf den Umgang mit OER zu proaktivem oder protektivem Verhalten führen, je nachdem ob ein Zugewinn oder ein Verlust an Reputation erwartet wird (vgl. Kursun et al. 2014, S. 22). So kann die Herausstellung der eigenen fachlichen Expertise und Erhöhung der eigenen Sichtbarkeit durch die Dokumentation dieser in OER ein Motivator für die Produktion und Bereitstellung von Bildungsmaterialien über digitale Kanäle und soziale Netzwerke sein (OECD 2007, S. 12). Lehrende können sich aber auch gegen die Bereitstellung von OER verwehren, weil sie fürchten, dass sie dann ihr Kapital, das Fachwissen, verlieren, da sie es kostenfrei und ggf. sogar schon kursgerecht aufbereitet anderen zur Verfügung stellen und damit die Konkurrenz für künftige, hochwertige Lehraufträge im entsprechenden Handlungskontext stärken (vgl. Mayrberger und Hofhues 2013, S. 61). Um dies zu vermeiden und zugleich die eigene Sichtbarkeit zu erhöhen, können Mittelwege als Strategie genutzt werden, bei denen 
eigene Materialien zwar offen, aber nicht frei publiziert werden, indem z.B. auf die Auszeichnung des Materials mit entsprechenden Lizenzen verzichtet wird. So könnten Lehrende persönliche Reputation gewinnen, indem sie auf ihre Sachkompetenz verweisen und zugleich die Nachnutzung bewusst oder unbewusst boykottieren.

Affirmative Einstellungen bezüglich OER können durch positive Erfahrung beim Teilen (Freude über die Akzeptanz) und Einsatz von OER in der Lehre (Freude über innovatives, passgenaues Material) bestärkt werden (vgl. Otto 2020, S. 79f.). Eine hohe Qualität und Aktualität sowie gute Verfügbarkeit und technische und didaktische Aufbereitung wirken hier verstärkend (vgl. Mayrberger und Hofhues 2013, S. 61).

\subsection{Soziale Dimension - Dürfen}

Die soziale Dimension spiegelt das (Inter)Agieren von Produzierenden freier Bildungsmaterialien wider und findet im Spannungsfeld des eigenständigen Erstellens, der fachlichen Community sowie der Unterstützung durch Dritte statt.

In die soziale Dimension fallen z. B. generell selbstlose Motive zur Unterstützung Anderer durch die eigene Arbeit und der Wunsch nach Austausch mit Peers über die eigene Arbeit. Aber auch die wachsende Akzeptanz des Teilens (,,sharing is caring“) und teils auch Erwartung an ein Teilen durch Dritte (bspw. im Rahmen öffentlich finanzierter Projekte), fallen in diese Dimension (vgl. OECD 2007, S. $11 \mathrm{ff}$.). So ist die individuelle Bereitschaft zu teilen auch von einer passenden Kultur der Zusammenarbeit abhängig, die von sozialen Werten der Kollaboration und Reziprozität einer gemeinsamen Erarbeitung und wechselseitigen Verbesserung von Bildungsmaterialien - also einem Geben und Nehmen in einem gleichwertigen Verhältnis geprägt ist (vgl. Koschorreck 2018, S. 4f.).

Oft sind jedoch auch Unsicherheiten bezüglich der Möglichkeiten eines rechtssicheren und technischen Teilens von Inhalten und ein Mangel an Unterstützungsstrukturen zur Förderung der Erstellung und Nachnutzung von OER Gründe für die Zurückhaltung der Lehrenden (vgl. Braun 2008, S. 66; Deimann und Bastiaens 2010, S. 14f.). Sie wissen schlichtweg nicht, was sie dürften und es fehlen Zugriffsmöglichkeiten auf Tools und Infrastrukturen, die eine Produktion von Materialien mit hohem inhaltlichen und technischen Nachnutzungspotenzial und deren Bereitstellung für Dritte ermöglichen würden (vgl. Deimann und Bastiaens 2010, S. 15).

Für die Nutzenden von OER stellt sich auf der sozialen Ebene die Herausforderung, den Produzierenden und deren Expertise zu vertrauen und bereit zu sein, deren OER für die eigenen Kurse und Trainings zu nutzen. Durch ihre Nachfrage fördern sie wiederum die Verbreitung von OER in der Weiterbildungspraxis. Gleichwohl sollten Nutzende in ihrer Haltung nicht rein konsumorientiert ausgerichtet sein, sondern sich selbst als mitverantwortlich für eine Qualitätsprüfung beziehbarer Materialien sehen (vgl. Mayrberger und Hofhues 2013, S. 62) sowie auch ihre Adaptionen erneut unter offener Lizenz bereitstellen, damit auch diese im Sinne einer Open Culture weiter geteilt werden und damit einer „Mitnahmekultur“ (Ebersbach et al. 2011, S. 246f.) vorgebeugt wird.

Aus Sicht der Nutzerinnen und Nutzer sind zudem Faktoren wie eine schnelle Auffindbarkeit, freier Zugang oder auch Unterstützungsmöglichkeiten, Lernres- 
sourcen an eigene pädagogische Settings anzupassen, als positiv zu benennen (vgl. Mayrberger und Hofhues 2013, S. $64 \mathrm{f}$.).

Zusammenfassend betrachtet sind mit Blick auf die für einen aktiven Umgang mit OER zu motivierenden Lehrenden sowie deren Vernetzung in einer durch eine Open Culture geprägten Community, Aspekte des Könnens, Wollens und Dürfens der Einzelnen relevant, um Ansatz- und Unterstützungsmöglichkeiten für eine Open Educational Practice zu identifizieren, die sowohl zur Qualitätssicherung von OER beitragen als auch Lernende als Produzierende des Prozesses des lebenslangen Lernens fördern können (vgl. OPAL 2011, S. 12).

\section{Ableitung der eigenen Fragestellungen}

In Gegenstand und Fragestellungen knüpft der Beitrag an den Diskursstand zum Potenzial von OER für eine durch Offenheit gekennzeichnete (Weiter)Bildungspraxis an und nimmt in den Blick, welche Relevanz OER für Lehrende selbst haben sowie inwiefern sie sich an einer Erstellung und Nachnutzung von OER beteiligen (würden). Konkret wird danach gefragt, (1) welche Faktoren eine Etablierung einer Kultur des Teilens aus Sicht Lehrender in der Erwachsenen- und Weiterbildung beeinflussen und (2) wie sich das Nutzungsverhalten Lehrender in Bezug auf Informations- und Lerninhalte zu OER am Beispiel von wb-web darstellt.

Die Fragestellungen zielen konkreter darauf zu untersuchen, welche der oben genannten Aspekte der sachlichen, sozialen und persönlichen Dimensionen des Erstellens, Teilens und Nachnutzens von OER in der Weiterbildungspraxis für Lehrende besonders bedeutsam sind. Einerseits soll damit ein Beitrag zur Sichtbarmachung der Sichtweise der Lehrenden zu dem Potenzial von OER als Wegbereiter für eine Kultur des Teilens geleistet werden. Andererseits sollen mithilfe der Ergebnisse hemmende und förderliche Aspekte für die Etablierung einer Community der Nutzung von OER, die durch eine Kultur des Teilens geprägt ist, identifiziert sowie daraus Ableitungen für Unterstützungsmaßnahmen getroffen werden.

\section{Methodisches Vorgehen}

Zur Untersuchung der relevanten Faktoren wurde eine Online-Befragung Lehrender aus der Erwachsenenbildung durchgeführt und die so gewonnenen subjektiven Einschätzungen zum Können, Wollen und Dürfen im Umgang mit OER mit einer Evaluation des tatsächlichen Nutzungsverhaltens der Angebote zum Umgang mit OER auf dem Portal wb-web und der daraus erschließbaren Informationsinteressen und Lernbedarfe der Lehrenden zur Erstellung, zum Einsatz und zum Teilen von OER gerahmt. Für die Evaluation wurde ein quantitatives Vorgehen gewählt, um in der Breite differenzierte Aussagen unter Einschluss möglichst vieler Aspekte treffen zu können.

Als Zielgruppe der Befragung wurden Lehrende aus den verschiedenen institutionellen Kontexten und Themenbereichen der Erwachsenen- und Weiterbildung über den wb-web-Unterstützerkreis bzw. die Netzwerke der Universität Tübingen 
angesprochen. An der Befragung im Zeitraum von Dezember 2020 bis Januar 2021 haben $n=97$ Lehrende teilgenommen. Nähere Aussagen zur Zusammensetzung der Stichprobe sind nicht möglich, da bei der Erhebung keine personen- und berufsbezogenen Daten erfasst wurden, um die Niedrigschwelligkeit der Teilnahme zu erhöhen und die Befragung im Umfang möglichst kurz zu halten. Inhaltlich wurden aus den oben skizzierten theoretischen Grundlagen abgeleitete Aspekte der sachlichen, sozialen und persönlichen Dimensionen des Erstellens, des Teilens und Nachnutzens von OER anhand geschlossener Fragen in den Blick genommen. Es wurden Fragen gestellt zu den notwendigen Voraussetzungen für die Produktion von OER (sachliche Dimension), zu der eigenen Haltung der Lehrenden gegenüber OER (persönliche Dimension) und zu den Werten und Möglichkeiten, selbst erstellte OER anderen zur Verfügung zu stellen (soziale Dimension). Weiter wurden anhand einer abschließenden offenen Frage Wünsche an den künftigen Umgang mit OER in der Weiterbildungspraxis erbeten. Die Daten wurden deskriptiv anhand von Häufigkeitsverteilungen ausgewertet. Weiter wurden die Befragten auf Basis der Nutzungsaktivität als Variable in drei Gruppen der (1) regelmäßigen breiten Nutzung $(37,1 \%)$, (2) themenbezogenen gelegentlichen Nutzung (38,1\%), (3) zufälligen eher seltenen Nutzung $(24,7 \%)$ unterteilt, um den Einfluss des eigenen Umgangs mit OER auf die mit OER verbundenen Werthaltungen zu untersuchen.

Die Betrachtung des Nutzungsverhaltens auf wb-web im Umgang mit Informations- und Lerninhalten zu OER erfolgte anhand von thematischen Zugriffen im Zeitraum von Juli bis Dezember 2020. Über eine Analyse der Nutzungshäufigkeit und -dauer (= Anzahl und Dauer der Zugriffe) und Nutzungsverläufe (= Wege der Nutzenden in der Auseinandersetzung mit den Inhalten) lassen sich für die Lehrenden besonders relevante OER-Inhalte des Portals für die Gestaltung von offenen Bildungsmaterialien und Entwicklung von Lehrkompetenz im Umgang mit OER identifizieren.

\section{Ergebnisdarstellung}

Die Ergebnisse der Befragung zu dem Potenzial von OER für die Weiterbildungspraxis zeugen insgesamt betrachtet von einer hohen Sensibilität Lehrender im Umgang mit OER. So sind 82,5\% der Befragten mit der begrifflichen Bedeutung von OER vertraut. Jedoch ist der Begriff nicht für alle Befragten mit positiven Aspekten behaftet. So bringen zwar viele Lehrende OER mit Zugänglichkeit $(90,7 \%)$ und Kostenfreiheit $(82,5 \%)$ in Verbindung, jedoch nur 51,5\% mit Transparenz und 25,8\% mit Vertrauen, während 13,4\% gar Chaos als Aspekt nennen. In diesen Zuschreibungen spiegelt sich eine gewisse Skepsis der Befragten wider, deren Ursache in der wahrgenommenen Qualität verfügbarer OER liegen könnte. Zumindest stellt nach Eigenaussage der Lehrenden eine hohe Qualität das zentrale Merkmal für die Auswahl von OER dar $(84,5 \%)$. Weiter legen die Befragten in Bezug auf die Qualität von OER insbesondere Wert auf die Aktualität (72,2\%), Verständlichkeit (70,1\%) und Praxisnähe $(63,9 \%)$. Für rund die Hälfte der Lehrenden sind weiter die Wissenschaftlichkeit (53,6\%), die mediale Aufbereitung (46,4\%), die Anpassbarkeit $(46,4 \%)$ und die Transparenz bezüglich des Urheberrechts $(42,3 \%)$ sowie die Art 


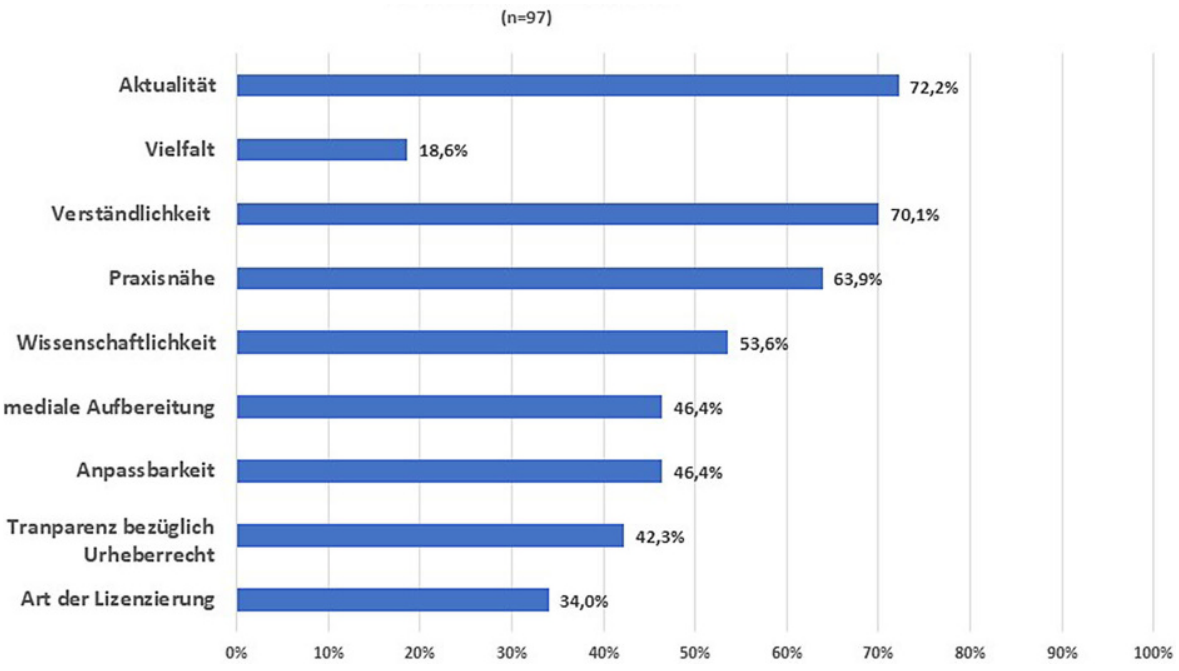

Abb. 2 Qualitätsaspekte für offene Bildungsmaterialien (OER). (Eigene Darstellung)

der Lizenzierung $(34,0 \%)$ entscheidend für die eigene Suche nach und den Einsatz von OER (vgl. Abb. 2).

Es ist somit nicht nur ein Bewusstsein der Lehrenden für OER vorhanden, sondern diese haben auch klare Erwartungen und Ansprüche an die Qualität von OER. Jedoch zeigt sich beim eigenaktiven Umgang mit OER in der Weiterbildungspraxis ein gemischtes Bild: Nur 37,1\% der Lehrenden nutzen regelmäßig und breit OER, $38,1 \%$ nutzen OER gezielt für einzelne Themen und 24,7\% nutzen eher selten OER, wenn sie bei der Suche nach generellen Informationen und Materialien zufällig auf OER stoßen. Weiter haben nur 22,7\% schon einmal Anderen Bildungsmaterialien als OER zur Verfügung gestellt, 43,3\% teilen Bildungsmaterialien auf Anfrage im eigenen Kollegium. Mögliche Erklärungen für die Diskrepanz zwischen Bewusstsein und Ansprüchen auf der einen Seite und eigenem Beitrag der Erstellung, des Teilens und des Nachnutzen von OER auf der anderen Seite, können in den Einschätzungen der Lehrenden zum (a) Können, (b) Wollen und (c) Dürfen als das Verhalten der Lehrenden im Umgang mit OER bedingende Dimensionen gefunden werden.

\subsection{Sachliche Dimension - Können}

Die Kompetenz der Lehrenden stellt für 55,7\% der Befragten die zentrale Voraussetzung für die Akzeptanz und Verbreitung von OER dar. Die befragten Lehrenden sehen sich rund zur Hälfte in der Lage, OER inhaltlich eigenständig zu erstellen $(48,5 \%)$. Hinsichtlich technischer Fragen für die Produktion von OER in für eine Nachnutzung geeigneten Formaten wünschen sich 29,9\% explizit Unterstützung. Noch größer ist der Unterstützungsbedarf bei rechtlichen Fragen. So fehlt den Befragten zu 57,7\% ein Einblick in rechtliche Grundlagen von OER. Die mangelnden Kenntnisse werden auch darin deutlich, dass nur ca. ein Drittel der Befragten korrekt beantworten kann, was die Lizenz CC BY SA 3.0 DE für die Nachnutzung, Modi- 
fikation und erneute Verfügbarmachung von Bildungsmaterialien bedeutet. 51,5\% sind in Bezug auf rechtliche Fragen im Umgang mit OER so unsicher, dass sie sich als Voraussetzung für die Verfügbarmachung und den Einsatz von OER Rechtssicherheit wünschen für den Fall, dass ihnen Fehler unterlaufen. Weiter werden fehlende Strukturen bemängelt, die ein Auffinden sowie die Auswahl von OER für die eigene Kursplanung unterstützen könnten (79,4\%).

\subsection{Persönliche Dimension - Wollen}

Die Haltung der befragten Lehrenden gegenüber OER ist tendenziell durch Offenheit gekennzeichnet. So sind 62,9\% der Ansicht, dass die Nutzung von OER zeitgemäß ist und 51,5\% begrüßen es, freie Bildungsmaterialien zu veröffentlichen. Die Ansicht steht in Verbindung mit den gesellschaftlichen Gegebenheiten. So sehen 60,8\% in der Digitalisierung einen Treiber für den Einsatz von OER in der Erwachsenenund Weiterbildung. Die Bereitschaft der Lehrenden zur Nutzung von OER in ihrer Lehre ist bei rund der Hälfte der Befragten gegeben. So haben 56,7\% schon mindestens einmal OER in ihrer Lehre eingesetzt und 52,6\% sind der Ansicht, dass sich ihre Lehre vielfältiger gestalten ließe, wenn es genug OER für ihr Fachgebiet gäbe. Die mit dem Einsatz von OER in der eigenen Lehre gemachten Erfahrungen hingegen bewertet nur ein Drittel der befragten Lehrenden als positiv. Fragt man nach den möglichen Ursachen dafür, geben die Lehrenden an, dass die von ihnen im Netz gefundenen OER ihren Qualitätsansprüchen nicht entsprechen, da diese für 59,8\% nicht wissenschaftlich, für 54,6\% nicht korrekt, für 44,3\% nicht aktuell und ebenso für 44,3\% didaktisch nicht gut aufbereitet sind. Die Bereitschaft, Bildungsmaterialien $\mathrm{zu}$ veröffentlichen und (im Kollegium) zu teilen, ist - wie oben dargestellt grundsätzlich bei zwei Drittel der Befragten gegeben. Dabei scheint weniger eine Bezahlung für eine Bereitstellung der eigenen Materialien als OER von Relevanz zu sein (nur 4,1\% legen Wert darauf), vielmehr fürchten 19,6\% der Befragten, dass andere ihre Ideen stehlen würden, 9,3\% sehen gar ihre Expertise und Auftragslage gefährdet. Auch die Angst vor inhaltlicher Kritik lässt 29,9\% der Befragten zögern, ihre Materialien frei zugänglich zu machen.

\subsection{Soziale Dimension - Dürfen}

Die Etablierung einer Kultur des Teilens hängt für die Befragten von der Bereitschaft der Lehrenden ab. Für 51,5\% der Befragten ist ein Teilen von OER nicht selbstverständlich und nur $15,5 \%$ haben bis dato OER schon mit CC-Lizenz frei verfügbar gemacht.

Während für 4,1\% ein Teilen grundsätzlich nicht infrage kommt, können sich das 38,1\% für die Zukunft vorstellen. Ihre eigene Bereitschaft zu Teilen knüpfen 63,9\% der Befragten an die Bereitschaft der anderen Lehrenden, ebenso OER zur Verfügung zu stellen. Sie wünschen sich zu 54,6\% mehr Kooperation mit Kolleginnen und Kollegen bezüglich der Erstellung und Nachnutzung von OER. Sie sind der Ansicht, dass durch soziale geteilte Werte eine Community von Lehrenden entstehen könnte, die durch einen regen thematischen Austausch zu lehrrelevanten Themen $(23,7 \%)$ und einen hohen Vernetzungsgrad $(35,1 \%)$ geprägt ist. Die Bildung solch 
einer Community ist nach Einschätzung der Befragten jedoch nicht nur von der Kompetenz, Haltung und Bereitschaft Einzelner abhängig, vielmehr steht diese auch im Zusammenhang mit den gesellschaftlichen und institutionellen Gegebenheiten. Während auf gesellschaftlicher Ebene eher Trends wie die zunehmende Digitalisierung für 46,4\% wegweisend für eine höhere Akzeptanz von OER sein könnten, werden auf institutioneller Ebene Investitionen in Infrastrukturen gefordert, die ein Auffinden von OER unterstützen $(79,4 \%)$, geeignete Tools für die technische Gestaltung und Nutzung von OER umfassen $(29,9 \%)$ und einen Onlinespeicher für die Bereitstellung bieten $(53,6 \%)$.

Bringt man im nächsten Auswertungsschritt die erfragte Bereitschaft der Lehrenden zu Teilen mit deren Nutzungsaktivität in Verbindung, lässt sich ein Zusammenhang feststellen. So ist die Bereitschaft zu Teilen in der Gruppe der Lehrenden $(37,1 \%)$, die regelmäßig OER in der eigenen Lehre nutzen, mit 44,4\% am höchsten ausgeprägt. Die 38,1\% an Lehrenden, die OER gezielt für einzelne Themen nutzen, sind zu 27,0\% zum Teilen bereit, während die dritte Gruppe der 24,7\% der Lehrenden, die OER eher zufällig nutzen, wenn sie bei der Suche nach generellen Informationen und Materialien auf OER zurückgreifen, zu 91,7\% nicht dazu bereit sind, anderen Lehrenden eigene Bildungsmaterialien frei zur Verfügung zu stellen. Es scheint, dass Lehrende eher bereit sind, anderen OER zur Verfügung zu stellen, wenn sie selbst erfahren und vertraut mit dem Einsatz von OER sind.

Für diese These spricht auch das in der die Befragung ergänzenden Evaluation des Nutzungsverhaltens der Lehrenden bezüglich der OER-Angebote auf wbweb gewonnene Bild zur Nutzungshäufigkeit und -dauer. Das Nutzungsverhalten zeugt von einem hohen Interesse an Informations- und Lerninhalten, die Fragen der sachgerechten Gestaltung und Nachnutzung von OER thematisieren. So nimmt zum Beispiel die Seite „CC-Lizenzen im Überblick - welche Lizenz für welche Zwecke“, die den Charakter einer Handreichung hat, mit 7951 Zugriffen bei 119.658 Besuchen Platz vier der meistbesuchten Seiten in der Rubrik Medien im Untersuchungszeitraum von Juli 2020 bis Dezember 2020 ein. Die Verweildauer von 89-135s lässt dabei auf eine aktive Rezeption der präsentierten Informationen schließen. Betrachtet man die weiteren Nutzungsverläufe, lässt sich feststellen, dass 71,8\% der in wbweb verbleibenden Nutzenden auf interne Seiten wechseln, die weitere rechtliche Fragen $(35,8 \%)$ vertiefen sowie auf inhaltliche und technische Gestaltungsfragen zu OER (36\%) eingehen. Auch die Abrufhäufigkeit des Wissensbausteins „Open Educational Resources - Wie frei lizenzierte Materialien der Weiterbildungspraxis helfen“, der zur Erklärung von Hintergründen, Begriffen und Einsatzmöglichkeiten offener Bildungsmaterialien dient, spricht für den Bedarf der Lehrenden, sich gezielt und vertieft mit den zum Umgang mit OER angebotenen Informationen rechtlicher sowie (medien)didaktischer Art vertraut machen zu wollen. Der Wissensbaustein wurde im Untersuchungszeitraum 591 Mal abgerufen. Damit liegt er in der Rubrik Medien auf Platz sieben, der in Summe 11.479 Abrufe im Untersuchungszeitraum verzeichnet.

Einen Hinweis auf den Bedarf der Lehrenden, das eigene Können im Umgang mit OER weiterzuentwickeln, geben die Zugriffszahlen auf den EULE Lernbereich von wb-web und die darin angebotenen Lernpfade für die Aneignung pädagogischpsychologischen Wissens und den Erwerb handlungsrelevanter Fähigkeiten der Kon- 
zeption, Umsetzung und Evaluation von Lehr-Lernangeboten. Auch hier fanden die beiden zu OER verfügbaren Lernpfade eine hohe Resonanz. So wurde im Zeitraum von Juli 2020 bis Dezember 2020 der Lernpfad zum Auffinden und Einsetzen von OER 479 Mal aufgerufen und der Lernpfad zur Erstellung und Veröffentlichung von OER 763 Mal. Auch hier lässt die durchschnittliche Verweildauer von 28:43 min bzw. 37:36 min je Lernpfad (im Vergleich zu der auf alle verfügbaren Lernpfade bezogenen durchschnittlichen Verweildauer von 12:50 min) auf eine aktive und umfassende Auseinandersetzung mit den Lerninhalten und gestellten Aufgaben zum Nachvollzug und Transfer auf die eigene Weiterbildungspraxis schließen.

Zusammenfassend betrachtet lassen die Untersuchungsergebnisse auf ein hohes Interesse der Lehrenden an Informations-, Lern- und Austauschmöglichkeiten zum Umgang mit OER schließen. Das zeigen nicht nur die hohen Nachfragezahlen der auf wb-web und im Lernbereich EULE enthaltenen Angebote zu dem Thema, sondern auch die Einschätzungen der Lehrenden zum Potenzial von OER für die Weiterbildungspraxis. Weiterführend lassen sich aus den Befragungsergebnissen auch Faktoren identifizieren, die Knackpunkte für die Erstellung, das Teilen und die Nachnutzung von OER in der Weiterbildungspraxis darstellen und in Maßnahmen für eine gezielte Förderung des Einsatzes von OER münden sollten. Drehund Angelpunkt einer aktiven Nutzung von OER sind dabei qualitativ hochwertige Bildungsmaterialien, die leicht zugänglich und rechtlich abgesichert zur Verfügung stehen. Damit solche Bildungsmaterialien den Weg für eine Etablierung einer Open Culture bereiten können, sind Investitionen in das Können, Wollen und Dürfen sowie in rahmende Unterstützungsfaktoren notwendig. Während es den Lehrenden mit Blick auf das Können nicht an pädagogischem Wissen und didaktischen Fähigkeiten zur Gestaltung von praxisrelevanten OER mangelt, herrscht bei rechtlichen und technischen Fragen eine hohe Unsicherheit. So besteht ein zentraler Erfolgsfaktor für die Nachnutzung und Verbreitung von OER in der Weiterbildungspraxis in der Unterstützung der Lehrenden beim Aufbau OER-spezifischen Wissens zur Produktion qualitativ hochwertiger und flexibel nachnutzbarer OER sowie dessen sachgerechter Adaption für weitere Anwendungsfälle. Hinsichtlich der persönlichen Bereitschaft, OER zu erstellen und zu teilen, ist die Gruppe der Lehrenden eher als heterogen einzustufen. Dabei wird augenscheinlich deutlich, dass die Haltung der Lehrenden mit dem Aufbau von Vertrauen in die Qualität von OER und insbesondere mit einer lizenz-rechtlichen Absicherung in Verbindung steht. Weiter scheinen Lehrende, die selbst OER nutzen, offen für einen Beitrag zur weiteren Verbreitung von OER zu sein. Hinsichtlich der sozialen Dimension des Teilens legen die befragten Lehrenden besonderen Wert auf ein Win-Win im kollegialen Austausch, wobei die zu investierenden Ressourcen für die Entwicklung von OER nicht zu unterschätzen sind sowie mehr rahmende Unterstützung durch Dritte für den Umgang mit OER, u.a. durch die Schaffung von Infrastrukturen gewünscht wird. Folgende Originalzitate zu den am Ende der Befragung wiederholt offen geäußerten Unterstützungswünschen bezüglich OER bringen dies folgendermaßen auf den Punkt:

Ich wünsche mir bezüglich OER vor allem die Möglichkeit, zentral OERs zu finden und eigene Materialien anderen auf zentraler Plattform zur Verfügung stellen zu können. 
Mehr Transparenz durch bessere Unterstützungsstrukturen, die Informationen zu rechtlichen und technischen Fragen der Gestaltung von OER bereitstellen, die Suchmöglichkeiten verbessern und ein Forum für den Austausch mit Lehrenden zu OER bieten.

\section{Diskussion und Fazit}

Die Ergebnisse der Untersuchung geben Auskunft zu verhaltensbedingenden Faktoren für einen aktiven Umgang mit OER in der Weiterbildungspraxis, die Anknüpfungspunkte für die Ableitung förderlicher Maßnahmen zur Etablierung einer durch einen kollaborativen Informations- und Wissensaustausch geprägten Community von Lehrenden der Erwachsenen- und Weiterbildung, die sachliche, persönliche und soziale Dimensionen gleichermaßen berücksichtigen, bietet. Die Untersuchung leistet einen Beitrag zu dem Diskurs zum Nutzungspotenzial von OER in der Erwachsenenbildung im Speziellen und der Bildungspraxis im Allgemeinen, indem sie empirische Erkenntnisse liefert zu der Sichtweise der Lehrenden als ideal geeignete Akteure für eine Produktion und Verbreitung von in hohem Maße bedarfsgerechten OER. Dabei bestätigen die erzielten Befunde die bestehenden Annahmen zu der Relevanz der Qualität(ssicherung) der Produktion und der Verwendung von OER um den Ansatz der Open Education zu beleben (vgl. UNESCO 2020, S. 24; Zawacki-Richter und Mayrberger 2017, S. 18) ebenso wie die Notwendigkeit eines kulturellen und strukturellen Wertewandels im Umgang mit Bildungsmaterialien, der ein flexibles Wachstum anstelle von starren Traditionen befördert (vgl. Ossiannilsson et al. 2016, S. 163; Powell 2015, S. 379). Die Ergebnisse reichen jedoch mit Blick auf die konkreten Bedarfe der Qualitätssicherung und Herausforderungen der Etablierung einer Open Culture weiter und zeigen konkrete Stellschrauben für eine gelebte Offenheit in der Erwachsenen- und Weiterbildung auf. Diese bestehen in der Qualifizierung der Lehrenden im rechtlichen und technischen Umgang mit OER und der Schaffung von Infrastrukturen, die ein Auffinden und eine Verfügbarmachung von frei zugänglichen Bildungsmaterialien niedrigschwellig ermöglichen sowie eine Reziprozität des Handelns begünstigen und eine rechtliche Absicherung garantieren.

In der Aussagekraft unterliegen die Befunde jedoch einigen Limitationen, denen es mit weiterer Forschung zu begegnen gilt. In einer kritischen Reflexion der eigenen methodischen Vorgehensweise ist zunächst die kleine, nicht zufällig gezogene Stichprobe der Befragten zu nennen, deren Repräsentativitätsprüfung für die gesamte Berufsgruppe der Lehrenden in der Erwachsenen- und Weiterbildung aus forschungspragmatischen Gründen zudem außen vor gelassen wurde. Weiter erlaubt die vorliegende Datenqualität nur den rein deskriptiven Bericht von Häufigkeiten. Mittlere Tendenzen und Standardabweichungen zu einer differenzierten Abbildung eventueller - bei der heterogenen Zielgruppe (vgl. Martin et al. 2016) erwartbarer unterschiedlicher Sichtweisen und eventuellen Typisierung von OER-Usern, wie sie für Hochschullehrende von Weller et al. (2016) vorgenommen wurde, konnten nicht berechnet werden. Ebenso wenig konnten komplexere bivariate oder multivariate Verfahren zur Auswertung der Daten herangezogen werden, die empirischen Auf- 
schluss über Zusammenhänge oder gar Abhängigkeiten zwischen einzelnen Variablen bzw. Dimensionen hätten geben können (vgl. z. B. Raithel 2008). Anschließende Studien können dazu dienen, diese Desiderate aufzugreifen und mit einer umfassenderen, auf höheren Skalenniveaus basierenden Befragung eine größere Teilnehmendenzahl anzustreben, die neben Lehrenden aus der Erwachsenen- und Weiterbildung gegebenenfalls auch das pädagogische Personal der Hochschule und Schule miteinschließen könnte, um nicht nur für die Erwachsenenbildung repräsentative Befunde zu erzielen, sondern im Sinne einer inkludierenden Open Education (vgl. Wiley 2010, S. 16) darüber hinaus einen Vergleich der Einschätzungen über verschiedene Bildungskontexte hinweg vornehmen zu können. Weiter könnte es aufschlussreich sein, Befragungen zum Nutzungspotenzial von OER in Form einer Zeitreihenanalyse wiederholt durchzuführen, um unter anderem mögliche Zusammenhänge zwischen einer Weiterentwicklung von Wissen und Fähigkeiten Lehrender durch zwischenzeitlich eingesetzte Unterstützungsmaßnahmen und einer Veränderung der (sozialen) Werthaltungen der Lehrenden zu untersuchen. Schließlich gilt es kritisch zu diskutieren, inwiefern quantitative Verfahren überhaupt geeignet sind, um Erkenntnisse zu einer Kultur des Teilens und zum Thema Community Building zu gewinnen. Zur Rekonstruktion (impliziter) Haltungen und Thematisierung von geeigneten Kommunikations- und Interaktionsformen könnten (ergänzende) qualitative Untersuchungen in Form von Interviews oder Gruppendiskussionen einen fruchtbaren Ansatz bieten.

Implikationen für die Praxis bestehen in der bedarfsgerechten Förderung der heterogenen Berufsgruppe der Lehrenden in der Erwachsenen- und Weiterbildung durch Angebote zum Aufbau OER-spezifischen Wissens und der Schaffung von zentralen, institutionenübergreifenden Orten, über die OER verfügbar gemacht sowie bezogen werden können und die Räume für einen kollaborativen Wissensaustausch bereithalten (vgl. Camilleri und Ehlers 2011, S. 9; OPAL 2011, S. 12). „Es gilt den Übergang zur selbstverständlichen Nutzung [von OER] so facettenreich wie innovationsfördernd zu gestalten und dafür an (tragfähige) bestehende Praktiken anzuknüpfen“ (Mayrberger und Hofhues 2013, S. 66), was bei dem heterogenen Feld der Erwachsenen- und Weiterbildung eine besondere Herausforderung darstellt und nur unter dem Einbezug der konkreten Interessen und Bedarfe der Zielgruppe gelingen kann. Hierzu könnten das Informations- und Vernetzungsportal wb-web sowie der darin enthaltene Lernbereich EULE einen möglichen zentralen Ansatzpunkt zur Bildung einer kontext- und handlungsfeldübergreifenden Community Lehrender in der Erwachsenen- und Weiterbildung bieten und die dort schon bestehenden Informations- und Lerninhalte zu OER-relevanten Themen entsprechend bedarfsgerecht ausgebaut werden.

In dem vorliegenden Beitrag wurde das Potenzial von OER für die Weiterbildungspraxis untersucht und nach Erfolgsfaktoren für die Etablierung einer Kultur des Teilens bei Lehrenden der Erwachsenen- und Weiterbildung gefragt. Die erzielten Befunde geben nicht nur Auskunft über sachliche, persönliche und soziale Dimensionen, die das Verhalten von Lehrenden im Umgang mit OER beeinflussen, sie bieten zudem Anhaltspunkte für die aus Sicht der Lehrenden bedarfsgerechte Förderung der Erstellung, des Teilens und der Nachnutzung von qualitativ hochwertigen OER als Wegbereiter für die Etablierung einer durch eine offene Kultur geprägten 
Community des freien und inkludierenden Informations- und Wissensaustausches Lehrender in der Erwachsenen- und Weiterbildung.

Open Access Dieser Artikel wird unter der Creative Commons Namensnennung 4.0 International Lizenz veröffentlicht, welche die Nutzung, Vervielfältigung, Bearbeitung, Verbreitung und Wiedergabe in jeglichem Medium und Format erlaubt, sofern Sie den/die ursprünglichen Autor(en) und die Quelle ordnungsgemäß nennen, einen Link zur Creative Commons Lizenz beifügen und angeben, ob Änderungen vorgenommen wurden.

Die in diesem Artikel enthaltenen Bilder und sonstiges Drittmaterial unterliegen ebenfalls der genannten Creative Commons Lizenz, sofern sich aus der Abbildungslegende nichts anderes ergibt. Sofern das betreffende Material nicht unter der genannten Creative Commons Lizenz steht und die betreffende Handlung nicht nach gesetzlichen Vorschriften erlaubt ist, ist für die oben aufgeführten Weiterverwendungen des Materials die Einwilligung des jeweiligen Rechteinhabers einzuholen.

Weitere Details zur Lizenz entnehmen Sie bitte der Lizenzinformation auf http://creativecommons.org/ licenses/by/4.0/deed.de.

\section{Literatur}

Aschersleben, K. (1993). Welche Bildung brauchen Schüler? Vom Umgang mit dem Unterrichtsstoff. Bad Heilbrunn: Klinkhardt.

Blees, I., Deimann, M., Seipel, H., Hirschmann, D., \& Muuß-Merholz, J. (2015). Whitepaper Open Educational Resources (OER) in Weiterbildung/Erwachsenenbildung. Bestandsaufnahme und Potenziale 2015. Bertelsmann Stiftung. https://open-educational-resources.de/wp-content/uploads/WhitepaperOER-Weiterbildung-2015.pdf. Zugegriffen: 7. Febr. 2021.

BMBF, \& Preusse, S. (2020). Der vielleicht wichtigste Aspekt zur Qualität von OER ist die Zusammenarbeit an Materialien. BMBF. https://www.qualifizierungdigital.de/de/der-vielleicht-wichtigsteaspekt-zur-qualitaet-von-oer-ist-die-zusammenarbeit-an-materialien-5654.php. Zugegriffen: 4. März 2021.

Braun, B. (2008). Die Open Educational Resources Bewegung. Optionen und Hürden für Lehrende an deutschen Hochschulen. Masterarbeit. Universität Duisburg-Essen. http://archiv.ub.uni-heidelberg. de/volltextserver/8676/1/masterarbeit_barbara_braun.pdf. Zugegriffen: 4. März 2021.

Camilleri, A. F., \& Ehlers, U.-D. (2011). Mainstreaming open educational practice recommendations for policy. Opal consortium. https://ulf-ehlers.net/list-of-onlineresources-from-eu-projects/. Zugegriffen: 7. Febr. 2021.

Deimann, M. (2019). Lernen mit Open Educational Resources. In H. Niegemann \& A. Weinberger (Hrsg.), Lernen mit Bildungstechnologien (S. 699-708). Wiesbaden: Springer.

Deimann, M., \& Bastiaens, T. (2010). Potenziale und Hemmnisse freier digitaler Bildungsressourcen eine Delphi-Studie. Zeitschrift für e-learning, 5(3), 7-18.

Ebersbach, A., Glaser, M., \& Heigl, R. (2011). Social web (2. Aufl.). Konstanz: UVK.

Ehlers, U.-D. (2013). Open learning cultures. A guide to quality, evaluation, and assessment for future learning. Wiesbaden: Springer.

Ehlers, U.-D. (2016). Open educational resources in Germany. In M. Fengchen, M. Sanjaya \& R. McGreal (Hrsg.), Open educational resources: policy, costs and transformation (S. 87-98). : UNESCO.

Friesinger, G., Herwig, J., Kroeger, O., \& Hrachovec, H. (2015). Ökonomien zwischen Öffnung und Begrenzung. In G. Friesinger, J. Herwig, O. Kroeger \& H. Hrachovec (Hrsg.), Open Culture: Ökonomien zwischen Öffnung und Begrenzung (S. 9-11). Wien: Edition Mono/Monochrom.

Kerres, M. (2019). Offene Bildungsressourcen und Open Education: Openness als Bewegung oder als Gefüge von Initiativen. Medienpädagogik - Zeitschrift für Theorie und Praxis der Medienbildung, $34,1-18$.

Koschorreck, J. (2018). Offene Bildungsressourcen - offene Bildungszugänge? Chancen und Herausforderungen. Magazin Erwachsenenbildung.at, 34, 05-1-05-9. https://erwachsenenbildung.at/magazin/ 18-34/05_koschorreck.pdf. Zugegriffen: 7. Febr. 2011.

Kursun, E., Cagiltay, K., \& Can, G. (2014). An investigation of faculty perspectives on barriers, incentives, and benefits of the OER movement in Turkey. International Review of Research in Open and Distri- 
buted Learning, 15(6), 14-32. http://www.irrodl.org/index.php/irrodl/article/view/1914. Zugegriffen: 31.01.2021.

Martin, A., Lencer, S., Schrader, J., Koscheck, S., Ohly, H., Dobischat, R., et al. (Hrsg.). (2016). Das Personal in der Weiterbildung: Arbeits- und Beschäftigungsbedingungen, Qualifikationen, Einstellungen zu Arbeit und Beruf. Bielefeld: wbv.

Mayrberger, K., \& Hofhues, S. (2013). Akademische Lehre braucht mehr „Open Educational Practices“ für den Umgang mit „Open Educational Resources“ - ein Plädoyer. Zeitschrift für Hochschulentwicklung, 8(4), 56-68.

OECD (2007). Giving knowledge for free. The emergence of open educational resources. Paris: OECD. http://www.oecd.org/edu/ceri/38654317.pdf. Zugegriffen: 28. Jan. 2021.

OPAL (2011). Beyond OER. Shifting focus to open educational practices. THE OPAL report 2011. https:// duepublico2.uni-due.de/rsc/viewer/duepublico_derivate_00025907/OPALReport2011_Beyond_ OER.pdf?page=1. Zugegriffen: 12. Febr. 2021.

Open Education Consortium (o.J.) About the open education consortium. https://www.oeconsortium.org/ about-oec/. Zugegriffen: 7. Febr. 2021.

Ossiannilsson, E., Zehra, A., \& Altinay, F. (2016). Transformation of teaching and learning in higher education towards open learning arenas: a question of quality. In P. Blessinger \& T. J. Bliss (Hrsg.), Open education: international perspectives in higher education (S. 159-177). Cambridge: Open Book Publishers.

Otto, D. (2020). Offene Bildungsmaterialien in der Schule für das Lehren und Lernen in der digitalen Welt: Cui bono? In K. Kaspar, M. Becker-Mrotzek, S. Hofhues, J. König \& D. Schmeinck (Hrsg.), Bildung, Schule, Digitalisierung (S. 77-82). Münster, New York: Waxmann.

Powell, A. B. (2015). Open culture and innvoation: integrating knowledge across boundaries. Media, Cultura \& Society, 37(3), 376-393.

Raithel, J. (2008). Quantitative Forschung. Ein Praxiskurs (2. Aufl.). Wiesbaden: Springer VS.

von Rosenstiehl, L., Molt, W., \& Rüttinger, B. (2005). Organisationspsychologie (9. Aufl.). Stuttgart: Kohlhammer.

Siebert, H. (2019). Didaktisches Handeln in der Erwachsenenbildung. Didaktik aus konstruktivistischer Sicht (8. Aufl.). Augsburg: Ziel.

UNESCO (2015). Position paper on education post-2015. https://unesdoc.unesco.org/ark:/48223/pf0000227 336/PDF/227336eng.pdf.multi. Zugegriffen: 7. Febr. 2021.

UNESCO (2020). Forum on the impact of open courseware for higher education in developing countries: final report. https://unesdoc.unesco.org/ark:/48223/pf0000128515_eng. Zugegriffen: 28. Jan. 2021.

Weller, M., de los Arcos, B., \& Farrow, R. (2016). Identifying categories of open educational resource users. In P. Blessinger \& T.J. Bliss (Hrsg.), Open education: international perspectives in higher education (S. 73-91). Cambridge: Open Book Publishers.

Wiley, D. (2010). Openness as catalyst for an educational reformation. Educause Review, 45(4), 15-20.

Zawacki-Richter, O., \& Mayrberger, K. (2017). Qualität von OER. Internationale Bestandsaufnahme von Instrumenten zur Qualitätssicherung von Open Educational Resources (OER) - Schritte zu einem deutschen Modell am Beispiel der Hamburg Open Online University. Universität Hamburg. https:// www.synergie.uni-hamburg.de/media/sonderbaende/qualitaet-von-oer-2017.pdf. Zugegriffen: 7. Febr. 2021.

Publisher's Note Springer Nature remains neutral with regard to jurisdictional claims in published maps and institutional affiliations. 